\title{
A Decomposition Theorem for $T$-Indistinguishability Operators. The Continuous Strict Archimedean Case
}

\author{
D. Boixader \\ Sec. Matemàtiques i Informàtica \\ ETS Arquitectura del Vallès \\ Universitat Politècnica de Catalunya (UPC) \\ Sant Cugat del Vallès, Catalonia, Spain, EU \\ dionis.boixader@upc.edu
}

\author{
J. Recasens \\ Sec. Matemàtiques i Informàtica \\ ETS Arquitectura del Vallès \\ Universitat Politècnica de Catalunya (UPC) \\ Sant Cugat del Vallès, Catalonia, Spain, EU \\ j.recasens@upc.edu
}

\begin{abstract}
In this paper we study the relationship between the cuts of a $T$-indistinguishability operator and the t-norm $T$ chosen to define fuzzy transitivity. The key result is, strict Archimedean t-norms provide equivalence relations as their zero cuts, and that property characterizes such t-norms. As a consequence, a decomposition theorem for such $T$-indistinguishabilities is proved.
\end{abstract}

Keywords-fuzzy relation; T-indistinguishability operator; generating family; t-norm; strict Archimedean t-norm

\section{INTRODUCTION}

Fuzzy relations have played a crucial role in fuzzy reasoning for years. The most important family of relations in both the classical and the fuzzy domains is, perhaps, that of equivalence relations. It provides a basis for many mathematical and Artificial Intelligence techniques and theories, ranging from the construction of the set $Q$ of all rational numbers in classical mathematics, to the similaritybased or the case based reasoning (CBR) in AI.

An indistinguishability operator with respect to a t-norm $T$, or a $T$-indistinguishability for short, is a fuzzy relation which aims at extending the standard concept of equivalence relation to the fuzzy framework. Although there is not a unique way of doing so, such relational structures are overwhelmingly accepted among researchers as the most natural way forward in that pursuit. $T$-indistinguishabilities appear in the literature under many different names, such as Similarity Relations [1], Likeness, Equality Relations or, simply, Fuzzy Equivalence Relations (FER) [2].

A classical equivalence relation is a reflexive, symmetric and transitive relation. The fuzzy version of both reflexivity and symmetry is straightforward. Fuzzy transitivity, though, requires the use of some t-norm $T$, because defining transitivity involves the and logical connective, and t-norms are the standard and fuzzy connectives.

Definition 1.1: A t-norm is an operation on the unit interval which is associative, commutative and satisfies the boundary conditions $T(x, 0)=0$ and $T(x, 1)=x$ for all $x$ and $y$ in $[0,1]$.
Also, we will generally assume within this paper that the tnorm $T(x, y)$ is continuous and Archimedean [3]. In practice, every continuous Archimedean t-norm is isomorphic to the sum of positive real numbers, bounded or unbounded. According to Ling's theorem [3], such t-norms can be obtained by means of some non-increasing continuous function $f:[0,1] \rightarrow[0, M]$ via $T(x, y)=f^{[-1]}(f(x)+f(y))$ for all $x$ and $y$ in $[0,1]$. The function $f$ is called an additive generator of $T$. If $M=f(0)=\infty$ then we say that $T$ is strict (example: $T=P R O D$, the standard product of real numbers restricted to the unit interval). Otherwise, $M=f(0)<\infty$ is said to be nonstrict $\quad(T=L U K, \quad$ the Lukasiewicz t-norm, i.e. $T(x, y)=\max \{1-x-y, 0\}$, is the standard example).

NOTE: being Archimedean or strict is not restricted to continuous t-norms only. Both conditions may be independently defined for general t-norms. We present it in this way for the sake of simplicity.

Definition 1.2 A fuzzy relation $E$ on a universe $X$, i.e. a mapping $R: X \times X \rightarrow[0,1]$ is:

1.2.1 Reflexive, if $E(x, x)=1$ for all $x$ in $[0,1]$.

1.2.2 Symmetric, if $E(x, y)=E(y, x)$ for all $x, y$ in $[0,1]$.

1.2.3 Transitive with respect to a given t-norm $T$, or $T$ transitive, if $T(E(x, y), E(y, z)) \leq E(x, z)$ for all $\mathrm{x}, \mathrm{y}$ and $\mathrm{z}$ in $[0,1]$.

Although every t-norm $T$ may be regarded as a suitable extension of the logical and to the multi-valued setting, each single choice of $T$ has an effect on the behavior of $T$ transitivity, and thus on the whole structure of the $T$ indistinguishability operator $E$.

One way in which two $T$-indistinguishability operators may differ is regarding their associated sets of $a$-cuts. The concept of $a$-cut is standard to the Fuzzy Sets theory [4,5]. When applied to fuzzy sets, $a$-cuts provide families of nested classical sets, ordered according to the standard order $\leq$ in $[0,1]$.

Supported by Spanish DGI research project TIN2009-07235 
Definition 1.3 (a-cut). Let $A$ be a fuzzy set on $X$. For every a in $[0,1]$ the (classical) sets:

$$
\begin{aligned}
& C_{[a]}(x)=\{y \in X: A(x) \geq a\} \\
& C_{(a)}(x)=\{y \in X: A(x)>a\}
\end{aligned}
$$

are called the $a$-cut, and the strict $a$-cut of A, respectively.

Some aspects of the $a$-cuts of fuzzy equivalence relations have been studied in [6]. More specifically, our goal in this paper is to investigate the effect of the chosen t-norm $T$ on the $a$-cuts of $E$.

Let $\mathrm{E}$ be a $T$-indistinguishability operator on a set $X$. Since a fuzzy relation $E$ is simply a fuzzy set on $X \times X$ then according to definition 1.2 the $a$-cuts of $E$ are the sets:

$$
\begin{aligned}
& C_{[a]}=\{(x, y) \in X \times X: E(x, y) \geq a\} \\
& C_{(a)}=\{(x, y) \in X \times X: E(x, y)>a\}
\end{aligned}
$$

But classical relations are classical subsets of $X \times X$, so that we may think of the relational $a$-cuts $C_{[a]}$ and $C_{(a)}$ of $E$ as the classical relations:

$$
\begin{aligned}
& R_{[a]}(x, y)= \begin{cases}1 & \text { if } E(x, y) \geq a \\
0 & \text { otherwise }\end{cases} \\
& R_{(a)}(x, y)= \begin{cases}1 & \text { if } E(x, y)>a \\
0 & \text { otherwise }\end{cases}
\end{aligned}
$$

The natural question to ask at this point is whether the cut relations $R_{[a]}$ and $R_{(a)}$ are or are not classical equivalence relations. And the answer is, it depends on the chosen t-norm $T$.

Is has been known since the early days of Fuzzy Logic [1] that, if $T=M I N$ (a non Archimedean though continuous tnorm), the $a$-cuts of all $T$-indistinguishability operators - also known in this case as Similarity Relations- are transitive, and thus classical equivalence relations.

It is also known that continuous Archimedean t-norms such as $T=L U K$ or $T=P R O D$ do not generally provide classical transitive relations as their $a$-cuts, but only reflexive and symmetric ones (the so-called Proximity Relations).

However, the strict 0 -cut play a crucial role in relation to the t-norm as it will be shown in Section 3. Under some general conditions, it is proved that only strict Archimedean t-norms provide equivalence relations as their strict 0 -cuts. Developing further on this idea, Section 4 presents a decomposition of such $T$-indistinguishabilies based on the underlying classical equivalence relation.

\section{RELATIONAL CUTS: PRELIMINARY ASPECTS}

We present some elementary properties about the $a$-cuts of a $T$-indistinguishability operator. Only in this section we will assume $T$ is a general t-norm.
We begin with the trivial cuts, which are the 0 and 1-cuts, and the strict 1-cut. Then:

Proposition 2.1 Let E be a $T$-indistinguishability operator.

2.1.1 $R_{[0]}=1$ (the total relation)

2.1.2 $R_{(1)}=0$ (the empty relation)

$2.1 .3 R_{[1]} \neq 0\left(R_{[1]}\right.$ is non-empty)

Proof. 2.1.1 and 2.1.2 are obvious. 2.1.3 is a consequence of $E$ being reflexive.

Lemma 2.2 Let $E$ be a $T$-indistinguishability operator. If $R_{[1]}(x, y)=1$ then $E(x, z)=E(y, z)$ for all $x, y$ and $z$ in $X$.

Proof. $R_{[1]}(x, y)=1$ means that $E(x, y)=E(y, x)=1$

Since $E$ is $T$-transitive

$$
\begin{aligned}
& E(y, z)=T(E(x, y), E(y, z)) \leq E(x, z) \text { and } \\
& E(x, z)=T(E(y, x), E(x, z)) \leq E(y, z)
\end{aligned}
$$

Proposition 2.3 Let $E$ be a reflexive and symmetric fuzzy relation on $X$. Then the following are equivalent statements:

2.3.1 There exists a t-norm $T$ such that $E$ is a $T$ indistinguishability operator on $X$.

2.3.2 $R_{[1]}$ is an equivalence relation such that $E(x, z)=E(y, z)$ if $R_{[1]}(x, y)=1$ for all $x, y$ and $z$ in $X$.

Proof. $[\Rightarrow$ ] Lemma 2.2 provides for the second part of 2.3.2. We only need to prove the transitivity of $R_{[1]}$. Note that $R_{[1]} \neq 0$ (Proposition 2.1.3). For any $x, y$ and $z, R_{[1]}(x, y)=1$ and $R_{[1]}(y, z)=1$ are equivalent to $E(x, y)=1$ and $E(y, z)=1$.

Since $E$ is $T$-transitive,

$$
1=T(1,1)=T(E(x, y), E(y, z)) \leq E(x, z)
$$

so that $R_{[1]}(x, z)=1$.

$[\Leftarrow] E$ is transitive at least with respect to the drastic tnorm $Z$, a non-continuous t-norm which is defined as

$$
Z(x, y)=\left\{\begin{array}{l}
x \text { if } y=1 \\
\mathrm{y} \text { if } \mathrm{x}=1 \\
0 \text { otherwise }
\end{array}\right.
$$

The a-cuts of a T-indistinguishability operator with $0<a<1$ define reflexive and symmetric but, in general, non transitive classical relations.

Proposition 2.4 Let E be a T-indistinguishability operator. For any $0<a<1$, the relations $R_{[a]}$ and $R_{(a)}$ are reflexive and symmetric. 
Proof. Fuzzy reflexivity and symmetry translates into classical ones. Straightforward.

The fact that, in general, the cut relations are not transitive is a consequence of $T(a, a)<a$, which is standard behavior to any Archimedean t-norm, such as the product or the Lukasiewicz t-norms. Thus, even if $E(x, y) \geq a$ and $E(y, z) \geq a \quad$ (i.e. $\quad R_{[a]}(x, y)=1 \quad$ and $\quad R_{[a]}(y, z)=1 \quad$ ) Ttransitivity does not allow us to infer $E(x, z) \geq a$ (i.e. $\left.R_{[a]}(x, z)=1\right)$ which would be needed to guarantee transitivity.

Example 2.5 The indistinguishability $E(x, y)=1-|x-y|$ on $X=[0,1]$ is transitive with respect to the Lukasiewicz tnorm. For $x=0, y=0.5$ and $z=1$ we have that $E(0,0.5)=0.5$ and $E(0.5,1)=0.5$ but $E(0,1)=0$, so that $R_{[0.5]}(0,0.5)=1$ and $R_{[0.5]}(0.5,1)=1$, but $R_{[0.5]}(0,1)=0$. Note that $T(0.5,0.5)=0$

A notable exception to this rule is provided by $T=M I N$, a continuous but non Archimedean t-norm) As we have said before, it is a well known fact that the a-cuts of $M I N$ indistinguishabilities, more generally known as a Similarity Relations, are classical equivalence relations.

Proposition 2.6 Let $E$ be a $T$-indistinguishability operator with $T=M I N$. Then, for all a in $[0,1]$,

2.6.1 $R_{[a]}$ is an equivalence relation.

2.6.2 If $X$ is finite and $a<1, R_{(a)}$ is an equivalence relation.

Proof. Straightforward.

The t-norms which are ordinal sums [3] provide yet another exception to the rule, in the sense that some particular cuts $R_{\left[a_{1}\right]}, R_{\left[a_{2}\right]} \ldots$ are classical equivalence relations, while others are not. As with $T=M I N$, these are not Archimedean t-norms and so we do not concern ourselves with them within the scope of this paper.

\section{THE CONTINUOUS ARCHIMEDEAN CASE}

In this section we relate the character of the continuous Archimedean t-norm $T$ (i.e. its being either strict or non-strict) to the transitivity of the strict 0 -cut of a $T$-indistinguishability operator $E$.

For the rest of this section, we will assume $T$ to be an Archimedean continuous t-norm. The main result is as follows.

Proposition 3.1 Let $T$ be a continuous Archimedean t-norm, and $E$ a $T$-indistinguishability operator on a set $X$. If $T$ is strict, then $R_{(0)}$ is an equivalence relation.

Proof. $R_{(0)}$ is reflexive and symmetric (Proposition 2.4).

Transitivity of $R_{(0)}$. If $R_{(0)}(x, y)=1$ and $R_{(0)}(y, z)=1$ then $E(x, y)>0$ and $E(y, z)>0$. Since $E$ is $T$-transitive, and
$T$ is strict, it follows that $0<T(E(x, y), E(y, z)) \leq E(x, z)$ and therefore $R_{(0)}(x, z)=1$

Next example shows that instances of $T$ indistinguishabilities having equivalence relations as their strict 0 -cuts do happen with any possible t-norm. Thus, the reciprocal of proposition 3.1 does not hold in general.

Example 3.2 Let $E$ be a (classical) equivalence relation on a set $X$. Then, $E$ is a $T$-indistinguishability for any t-norm $T$, and $R_{(0)}=E$.

However, for a restricted class of $T$-indistinguishabilities, $R_{(0)}$ being an equivalence relation is a sufficient condition for the strictness of the Archimedean t-norm $T$. That class is defined by the two conditions:

3.3.1 $E(x, y)=1$ if, and only if, $x=y$, for all $x, y$ in $X$. ( $E$ is called a separating $T$-indistinguishability).

3.3.2 There exist $x \neq y \neq z \neq x$ in $X$, such that $E(x, z)=0$ and $E_{T}(E(x, z), E(x, y))=E(z, y)$

Condition 3.3.1 is a standard one. Condition 3.3.2 is closely related to the following characterization of strict continuous Archimedean t-norms.

Lemma 3.4 Let $T$ be a continuous Archimedean t-norm. The following statements are equivalent:

3.4.1 $T$ is strict.

3.4.2 $E_{T}(a, 0)=0$ for all $a>0$.

3.4.3 There exists $a$ with $1>a>0$ s.t. $E_{T}(a, 0)=0$.

Proof. Let $f$ be an additive generator of $T$. Remember that $T$ is strict if and only if $M=f(0)=\infty$, and also that $E_{T}(a, b)=I_{T}(a, b)=f^{[-1]}(f(b)-f(a))$ if $a \geq b$.

So if $T$ is strict, then

$E_{T}(a, 0)=\lim _{b \rightarrow 0} f^{[-1]}(f(b)-f(a))=f^{[-1]}(\infty)=0$ provided that $a>0$ (i.e. $f(a)<\infty$ ). This proves $3.4 .1 \Rightarrow 3.4 .2$

$3.4 .2 \Rightarrow 3.4 .3$ is obvious.

3.4.3 $\Rightarrow 3.4 .1 \quad E_{T}(a, 0)=f^{[-1]}(f(0)-f(a))=0$ implies that $M-f(a)=M$ Since $f(a) \neq 0$ because $1>a>0$, it must be $M=\infty$

Theorem 3.5 Let $T$ be an Archimedean t-norm, and $E$ a $T$ indistinguishability operator on $X$ satisfying conditions 3.3.1 and 3.3.2. Then $T$ is strict if, and only if, $R_{(0)}$ is an equivalence relation.

Proof. $[\Rightarrow]$ A consequence of proposition 3.1 
$[\Leftarrow]$ Let us consider such $x, y$ and $z$ as required in 3.3.2. $E(x, z)=0$, so $R_{(0)}(x, z)=0$. Condition 3.3.2 may be written as $E_{T}(E(x, z), E(x, y))=E_{T}(0, E(x, y))=E(z, y)$. We need to prove that $0<E(x, y)<1$ and $E(z, y)=0$ in order to be in the conditions 3.4 .3 of lemma 3.4 , which will prove that $T$ is strict.

But $E(x, y) \neq 1$ (condition 3.3.1), and also $E(x, y) \neq 0$ to avoid $1=E_{T}(0,0)=E(z, y)$ (also against condition 3.3.1). So it must be $0<E(x, y)<1$, and $R_{(0)}(x, y)=1$.

Then, since we are assuming $R_{(0)}$ to be an equivalence relation, it must be $R_{(0)}(y, z)=0$, and $E(z, y)=0$ accordingly. Otherwise, if $R_{(0)}(y, z)=1$ then transitivity and $R_{(0)}(x, y)=1$ would lead to $R_{(0)}(x, z)=1$ and $E(x, z)>0$, against condition 3.3.2 .

Corollary 3.6 Let $X=[0,1], T$ a continuous Archimedean t-norm and $E=E_{T} . T$ is strict if, and only if, $R_{(0)}$ is an equivalence relation.

Proof. $E=E_{T}$ satisfies conditions 3.3.1 and 3.3.2

Note that the equivalence relation splits the unit interval into two classes, namely $C_{1}=\{0\}$ and $C_{2}=(0,1]$, regardless the particular strict t-norm $T$ involved.

Although conditions 3.3.1 and 3.3.2 cannot be spared in theorem 3.5 , the fact still remains that the transitivity of $R_{(0)}$ is a feature essentially related to the strictness of the t-norm $T$, at least in the finite case.

Proposition 3.7 Let $X$ be a finite set, and $E$ a $T$ indistinguishability operator on $X . R_{(0)}$ is an equivalence relation, if, and only if, there exists a strict continuous Archimedean t-norm $T_{l}$ such that $E$ is also a $T_{1^{-}}$ indistinguishability operator.

Note that nothing is said about the original $T$, which may or may not be a strict t-norm.

Proposition 3.7 relies upon the following lemma concerning continuous Archimedean t-norms.

Lemma 3.8 Given $0<a<a_{1} \ldots \leq a_{n}<1$ there exists a continuous strict Archimedean t-norm $T_{l}$ such that $T\left(a_{i}, a_{j}\right) \leq a$ for all $i, j$.

Proof. Define an additive generator $f$ such that $f(a)=2 f\left(a_{n}\right)$ and $M=f(0)=\infty$

Proof. (Prop. 3.7) Lemma 3.8 together with $X$ being a finite set guaranties the existence of a continuous strict Archimedean t-norm $T_{1}$ such that $T_{1}(E(x, y), E(z, t)) \leq a=\min _{u, v \in X} E(u, v)$ and makes $E T_{1^{-}}$ transitive.
Next example shows that $X$ being finite is an essential condition.

Example 3.9 Let $X=(0,1]$ (i.e. the unit interval without 0$)$ and $E=E_{T}$ with $T=T_{L}$ (the Lukasiewicz t-norm). Then $R_{(0)}=1$, an equivalence relation indeed.

\section{DECOMPOSITION OF A $T$-INDISTINGUISHABILITY OPERATOR WITH $T$ STRICT}

In this section we develop further on the central idea from section III, namely, that $T$-indistinguishability operators with respect to strict Archimedean continuous t-norms do provide classical equivalence relations as their strict 0 -cuts.

The main result is that such fuzzy relations can be split into simpler fuzzy relations which are defined on smaller parts of $X$, and rebuilt from those afterwards in a way which reminds us of the Representation Theorem [7].

First, note that as a consequence of proposition 3.1, every $T$-indistinguishability with respect to a strict Archimedean continuous t-norm $T$ splits the set $X$ into a family $\left\{C_{i}\right\}_{i \in I}$ of equivalence classes, or clusters. This is common behavior to all classical equivalence relations, and it will allow us to prove the following theorem.

Theorem 4.1 Let $T$ be a strict Archimedean continuous tnorm. A fuzzy relation $E$ is a $T$-indistinguishability operator on $X$ if, and only if, there exists a partition $\left\{C_{i}\right\}_{i \in I}$ of $X$ and a family of $T$-indistinguishability operators $\left\{E_{i}\right\}_{i \in I}$ such that:

4.1.1 $E_{i}(x, y)>0$ iff $x$ and $y$ belong to the same $C_{i}$

4.2.2 $E(x, y)=\inf _{i \in I} E_{i}(x, y)$ for all $x, y$ in $X$.

Proof. $\quad[\Leftarrow]$ The infimum of $T$-indistinguishability operators is a $T$-indistinguishability operator [2].

$[\Rightarrow]$ Since $R_{(0)}$ is an equivalence relation (proposition 3.1 ), then we may consider the associated set of clusters $\left\{C_{i}\right\}_{i \in I}$ which is a partition of $X$.

For each cluster $C_{i}$ we define

$$
E_{i}(x, y)=\left\{\begin{array}{cc}
E(x, y) & \text { if } x \in C_{i} \text { and } y \in C_{i} \\
0 & \text { if } x \in C_{i} \text { and } y \notin C_{i} \\
1 & \text { if } x \notin C_{i} \text { and } y \notin C_{i}
\end{array}\right.
$$

It follows that $E(x, y)=\inf _{i \in I} E_{i}(x, y)$

So $T$-indistinguishability operators with respect to strict continuous Archimedean t-norms can be regarded as classical equivalence relations $R$ whose pairs $R(x, y)=1$ have been graded into $(0,1]$ while keeping the pairs $R(x, y)=0$ unchanged. The number of clusters (i.e. $|I|$, the cardinal of I) provides a measure of the complexity of $E$. 
A more standard measure of the complexity of an indistinguishability operator is its dimension. The concept of dimension arises from the Representation Theorem:

Theorem 4.2 [7] A fuzzy relation $E$ on a set $X$ is a $T$ indistinguishability operator if, and only if, there exists a family $\left\{h_{j}: X \rightarrow[0,1]\right\}_{j \in J}$ of fuzzy subsets of $X$ such that $E(x, y)=\inf _{j \in J} E_{T}\left(h_{j}(x), h_{j}(y)\right)$

Such families of fuzzy sets as $\left\{h_{j}: X \rightarrow[0,1]\right\}_{j \in J}$ are called generating families for obvious reasons, and the fuzzy sets $h_{j}$ are called generators of $E$. $E$ may have many such generating families, all of them differing in their number of generators (i.e. differing in $|J|$, the cardinal of $J$ ).

The dimension of $E$, which is noted $\operatorname{dim}(E)$, is the minimum $|J|$ of all, and a generating family having exactly $\operatorname{dim}(E)$ generators is called a basis of $E$ (see [2] for details).

Note that theorem 4.2 applies also to classical equivalence relations, which have generating families made up of classical, non fuzzy, subsets, dimension and basis. Next theorem establishes the relationship between the classical and the fuzzy dimensions.

Theorem 4.3 Let $T$ be a strict Archimedean continuous tnorm., and $E$ a $T$-indistinguishability operator on $X$. Then:

$$
\operatorname{dim}\left(R_{(0)}\right) \leq \operatorname{dim}(E) \leq \operatorname{dim}\left(R_{(0)}\right)+\max _{j \in J} \operatorname{dim}\left(E_{i}\right)
$$

\section{Proof.}

Before proceeding to prove theorem 4.3 we will show that neither the lower nor the upper bounds for $\operatorname{dim}(E)$ in [4.3.1] can be improved.

Example 4.4 (Lower bound) Consider $X=[0,1]$ and $E=E_{T}$ Then both $E$ and the associated $R_{(0)}$ are onedimensional and thus $\operatorname{dim}\left(R_{(0)}\right)=\operatorname{dim}(E)$

Example 4.5 (Upper bound) Consider $X=(0,3]$ and $C_{1}=(0,1], C_{2}=(1,2], C_{3}=(2,3]$ which form a partition of $X$. Let us define $E$ on $X$ as follows:

$$
E(x, y)=\left\{\begin{array}{cc}
E_{T}(x, y) \text { if } x \in C_{i} \text { and } y \in C_{i} \\
0 \quad \text { if } x \in C_{i} \text { and } y \notin C_{i}
\end{array}\right.
$$

Then $E$ is a $T$-indistinguishability on $X$ which cannot be generated with less than three fuzzy sets, so that $\operatorname{dim}(E)=3$.

The decomposition according to theorem 4.1 is

$$
E_{i}(x, y)=\left\{\begin{array}{cc}
E_{T}(x, y) \text { if } x \in C_{i} \text { and } y \in C_{i} \\
0 \quad \text { if } x \in C_{i} \text { and } y \notin C_{i} \quad i=1,2,3 . \\
1 \quad \text { if } x \notin C_{i} \text { and } y \notin C_{i}
\end{array} \quad\right.
$$

A basis for $E$ may be, for example, $h_{1}, h_{2}, h_{3}: X \rightarrow[0,1]$ as defined by

$$
h_{j}(x)=\left\{\begin{array}{l}
\mathrm{x} \text { if } x \in C_{j} \\
0 \text { if } x \notin C_{j}
\end{array} \quad j=1,2,3 .\right.
$$

Each $E_{i}$ has its own basis consisting in the only fuzzy set $h_{i}$, so $\operatorname{dim}\left(E_{i}\right)=1$ for $i=1,2,3$ and $\max _{j \in J} \operatorname{dim}\left(E_{i}\right)=1$

The strict 0 -cut is the classical equivalence relation

$$
R_{(0)}(x, y)= \begin{cases}1 & \text { if } x \in C_{i} \text { and } y \in C_{i} \\ 0 & \text { if } x \in C_{i} \text { and } y \notin C_{i}\end{cases}
$$

Obviously $C_{1}, C_{2}$ and $C_{3}$ are the equivalence classes or clusters of $R_{(0)}$ and therefore only two classical sets are needed in order to generate $R_{(0)}$. Two such sets may be, for example $g_{1}, g_{2}: X \rightarrow\{0,1\}$ defined by

$$
g_{i}(x)=\left\{\begin{array}{ll}
1 & \text { if } x \in C_{i} \\
0 & \text { if } x \notin C_{i}
\end{array} \quad i=1,2 .\right.
$$

(i.e. the characteristic functions of $C_{1}$ and $C_{2}$ as classical sets).

This example shows that

$$
3=\operatorname{dim}(E)=\operatorname{dim}\left(R_{(0)}\right)+\max _{j \in J} \operatorname{dim}\left(E_{i}\right)=2+1
$$

The following lemmata will be needed in order to prove theorem 4.3.

Lemma 4.6 Let $\left\{h_{j}: X \rightarrow[0,1]\right\}_{j \in J}$ be a basis of $E$. Then $\left\{g_{j}: X \rightarrow[0,1]\right\}_{j \in J}$ defined by $g_{j}(x)=\left\{\begin{array}{ll}1 & \text { if } h_{j}(x)>0 \\ 0 & \text { if } h_{j}(x)=0\end{array}\right.$ is a generating family of $R_{(0)}$.

\section{Proof. Straightforward.}

Lemma 4.7 Let $E$ be a $T$-indistinguishability operator on $X$, and $\left\{E_{i}\right\}_{i \in I}$ its decomposition according to theorem 4.3. Let $f_{i}$ be a generator of $E_{i}$ such that $f_{i}(x)=0$ for all $x \notin C_{i}$, and let $f_{j}$ be a generator of $E_{j}$ such that $f_{j}(x)=0$ for all $x \notin C_{j}$. Then $f=\max \left\{f_{i}, f_{j}\right\}$ verifies:
4.7.1 $E_{f}(x, y)=E_{f_{i}}(x, y)$ if if $x \in C_{i}$ and $y \in C_{i}$
4.7.2 $E_{f}(x, y)=E_{f_{j}}(x, y)$ if $x \in C_{j}$ and $y \in C_{j}$
4.7.3 $E_{f}(x, y)=0$ if $x \in C_{i} \cup C_{j}$ and $y \notin C_{i} \cup C_{j}$
4.7.4 $E_{f}(x, y) \geq E(x, y)$ otherwise. 
Proof (Theorem 4.3). The first inequality $\operatorname{dim}\left(R_{(0)}\right) \leq \operatorname{dim}(E)$ is a simple consequence of lemma 4.6.

We need to produce a generating system with exactly $\operatorname{dim}\left(R_{(0)}\right)+\max _{j \in J} \operatorname{dim}\left(E_{i}\right)$ elements in order to prove the second inequality.

Such a system may be build as the reunion of a basis of $R_{(0)}$, say $\left\{g_{m}: X \rightarrow\{0,1\}\right\}_{m \in M}$, and a set of generators of the fuzzy part of $E$ which are constructed as follows.

Let $\left\{f_{1}^{i}, f_{2}^{i}, \ldots\right\}$ be a basis of $E_{i},\left\{f_{1}^{j}, f_{2}^{j}, \ldots\right\}$ a basis of $E_{j}$, and so on. Suppose we have ordered the set $I$ according to the dimensions of $E_{i}$, i.e. $i \leq j$ iff $\operatorname{dim}\left(E_{i}\right) \geq \operatorname{dim}\left(E_{j}\right)$. Let us assume that $i$ is the first element, $j$ the second, and so on, so that $\left\{f_{1}^{i}, f_{2}^{i}, \ldots\right\}$ has more elements than $\left\{f_{1}^{j}, f_{2}^{j}, \ldots\right\}$ etcetera.

Then, according to lemma 4.8, the fuzzy sets $f_{1}=\max \left\{f_{1}^{i}, f_{1}^{j}, \ldots\right\}, f_{2}=\max \left\{f_{2}^{i}, f_{2}^{j}, \ldots\right\}$, etcetera are generators of $E$ such that $E(x, y)=\inf _{i=1,2, \ldots} E_{f_{i}}(x, y)$ for all $x, y$ sharing the same cluster $C_{k}$, and $E(x, y) \leq \inf _{i=1,2, \ldots} E_{f_{i}}(x, y)$ if they belong to different clusters. In the latter case, though, $E(x, y)=0$ and $\left\{g_{m}\right\}_{m \in M}$ (the basis of $R_{(0)}$ ) generates $E$.

We have proved that $\left\{g_{m}\right\}_{m \in M} \cup\left\{f_{1}, f_{2}, \ldots\right\}$ is a generating system for $E, \quad$ and thus $\operatorname{dim}(E) \leq\left|\left\{g_{m}\right\}_{m \in M} \cup\left\{f_{1}, f_{2}, \ldots\right\}\right|=\operatorname{dim}\left(R_{(0)}\right)+\max _{i \in I} \operatorname{dim}\left(E_{i}\right)$

\section{CONCLUSIONS}

Although it has been known for years that MINindistinguishability operators provide classical equivalence relations as their $a$-cuts, not much have been said about the relationship between the structure of these cuts and the type of t-norm involved in the definition of $T$-transitivity for other $\mathrm{t}$ norms.

In this paper we have thoroughly studied a class of t-norms, namely the strict continuous Archimedean t-norms. The main result regarding such t-norms is that strict 0 -cuts provide equivalence relations, and to some extent this behavior characterizes them.

Our next step will be the study of another important class of continuous t-norms: the ordinal sums. They are middle way between the continuous Archimedean, additively generated tnorms, such as $T=P R O D$ or $T=L U K$, and the minimum tnorm $T=M I N$, so mixed behavior is expected.

\section{REFERENCES}

[1] L. A. Zadeh, "Similarity relations and fuzzy orderings," Information Sciences, 3, 1971, pp. 177-200.

[2] Recasens, J. Indistinguishability Operators. Modelling Fuzzy Equalities and Fuzzy Equivalence Relations Series: Studies in Fuzziness and Soft Computing, Vol. 260. 2011. Springer Verlag. ISBN 978-3-642-16221-3.

[3] B.Schweizer, A.Sklar "Probabilistic Metric Spaces" North Holland, Amsterdam, (1983)

[4] G. J. Klir and Bo Yuan, Fuzzy sets and fuzzy logic. Theory and applications. New Jersey: Prentice Hall, 1995, ch. 3.

[5] Radim Bělohlávek, "Cutlike semantics for fuzzy logic and its applications", International Journal of General Systems, 32:4, 305-319

[6] Jirí Mockor, "Cut systems in sets with similarity relations", Fuzzy Sets and Systems 161 (2010) 3127-3140

[7] L. Valverde, "On the Structure of F-indistinguishability Operators ", Fuzzy Sets and Systems, 17, 1985, pp.313-328. 